\title{
The frequency and costs of intravitreal therapy agents in retinal diseases
}

\author{
Mahmut Atum ${ }^{\circledR}$, Burçin Çakır ${ }^{\circledR}$, İsa Yuvacı ${ }^{\circledR}$, Erkan Çelik ${ }^{\circledR}$, Gürsoy Alagöz ${ }^{\circledR}$ \\ Department of Ophthalmology, Sakarya University Education and Research Hospital, Sakarya, Turkey
}

\begin{abstract}
BACKGROUND: The aim of this study is to analyze the numbers and the economic burden of intravitreal anti-VEGF agents and intravitreal dexamethasone (IVD) implants administered to patients with diabetic retinopathy (DR), age-related macular degeneration (AMD) and retinal vein occlusion (RVO).

MATERIAL AND METHODS: The retrospective case-control study included 1525 patients diagnosed with DME, neovascular AMD and RVO, and received intravitreal anti-VEGF and IVD between January 2016 and December 2018. Intravitreal anti-VEGF administration was performed within the framework of the Pro Re Nata (PRN) regimen. The prices of anti-VEGF agents and IVDs were calculated on the average of the prices in the relevant year. RESULTS: The total number of intravitreal injections in 3 years was 5864 . During the 3 -year follow-up, on average, ranibizumab (Lucentis) was applied $3.56 \pm 2.25$ times, alfibercept (Eylea) was applied $3.31 \pm 2.16$ times, and IVD (Ozurdex) was applied $1.70 \pm 0.83$ times. The anti-VEGF numbers in 2016, 2017 and 2018 were 1997, 1801, 2066, respectively. In total, the 3-year drug cost was 3,587,812.44 USD.

CONCLUSIONS: The economic burden of intravitreal anti-VEGF and IVD treatment for retinal diseases is so important to developing countries such as Turkey. The economic burden created by anti-VEGF agents and IVDs in Turkey will reduce in a serious sense, and the legal concerns of physicians will decrease thanks to the decision taken by the Turkish Medicines and Medical Devices Agency (TMMDA).
\end{abstract}

KEY WORDS: retinal diseases; anti-VEGF; economic burden; diabetic retinopathy; age-related macular degeneration; retinal vein occlusion

Ophthalmol J 2020; Vol. 5, 55-59

\section{INTRODUCTION}

Diabetic retinopathy (DR), age-related macular degeneration (AMD) and retinal vein occlusion (RVO) are the most important causes of vision loss [1]. It is estimated that these three retinal diseases will gradually increase all over the world [2]. Diabetic macular edema (DME) due to $\mathrm{DR}$, neovascular AMD and RVO-induced macular edema (ME) are the main reasons for decreased vision. In recent years, intravitreal anti-vascular endothelial growth factor (anti-VEGF) agents have been used frequently in the treatment of
ME due to these three retinal diseases [3-5]. The most commonly used anti-VEGF agents are aflibercept (Eylea, Regeneron Pharmaceuticals, Tarrytown, New York, USA and Bayer Healthcare Pharmaceuticals, Berlin, Germany), bevacizumab (Avastin, Genentech, Inc., South San Francisco, CA, USA) and ranibizumab (Lucentis; Genentech, South San Francisco, CA, USA). Intravitreal dexamethasone implant (IVD; Ozurdex; Allergan Inc, Irvine, CA, USA) is also used for treatment of ME. The efficiencies of all these agents were similar in the studies conducted [5-7]. 
The widespread use of anti-VEGF agents causes a serious economic burden. In a study, it was reported that 2.1 million anti-VEGFs were used in the United States in 2013 and the approximate cost of this amount was more than 2.3 billion dollars [8]. Studies have shown that bevacizumab is 30-40 times cheaper than other agents and it is cost-effective [9-11]. As in many countries, there is no indication for intraocular use of bevacizumab in Turkey. Legal and ethical problems related to this issue limit the use of bevacizumab by ophthalmologists. Turkish Medicines and Medical Devices Agency (TMMDA) took a new decision on December 28, 2018, and according to this decision, it is stated that 3 dose bevacizumab should be used in patients who need anti-VEGF, and then other anti-VEGF agents can be used only in resistant and/or unresponsive cases [12]. It is estimated that this application aims to reduce the economic burden of anti-VEGF agents.

The aim of this study is to analyze the numbers and the economic burden of intravitreal anti-VEGF agents and IVD implants administered to patients with DME, AMD and RVO between January 2016 and December 2018.

\section{MATERIAL AND METHODS}

This retrospective study included 1525 patients who were diagnosed with DME, neovascular AMD and RVO, and received intravitreal anti-VEGF between January 2016 and December 2018. The study was conducted in the Retina Clinic of Sakarya University Education and Research Hospital. Approval for the study was obtained from the local ethics committee (Sakarya University Medical Faculty Ethics Committee, 15.02.2019/38). A detailed ophthalmologic examination was performed in all patients who were referred to the ophthalmology clinic, referred to the retinal unit, and followed up. In all patients, the best corrected visual acuity with Snellen chart, intraocular pressure measurement with Goldmann applanation tonometer, and anterior and posterior segment examination with a slit-lamp bio microscopy were performed. In addition, in all patients, a central foveal thickness with optical coherence tomography device (OCT, Cirrus HD OCT, Carl Zeiss Meditec, Dublin, CA, USA) and a fundus photograph and fluorescein angiography with fundus fluorescein angiography device (FFA, Canon Sales Co., Inc., Chiba, Japan) were performed. As a result of these measurements, intravitreal anti-VEGF treatment was applied to the patients in need of anti-VEGF by the ophthalmologist in the operating room conditions. Intravitreal anti-VEGF administration was performed within the framework of the PRN protocol adopted in EURETINA guidelines [13]. According to this protocol, all naive patients were given monthly injections in OCT until the intraretinal/subretinal fluid passed and anti-VEGF was administered when needed. In addition, anti-VEGF changes were performed or dexamethasone implants were used in patients who did not respond to treatment.

The prices of anti-VEGF agents and IVDs were calculated on the average of the prices in the relevant year (the year anti-VEGF administered). The calculations made over Turkish Lira (TL) were converted to US dollar (USD) by basing on the average dollar exchange rate of the Central Bank of the Republic of Turkey in the relevant year. The average price of Lucentis in 2016, 2017, 2018 was 704.16 USD (2128.97 TL), 644.58 USD (2352.72 TL), 505.75 USD (2437.83 TL), respectively. In the same years, the price of Eylea was 592.54 USD (1791.5 TL), 496.45 USD (1812.05 TL) and 415.99 USD (2005.16 TL), respectively. Furthermore, the price of Ozurdex was 665.96 USD (2013.48 TL), 609.57 USD (2224.93 TL), 529.92 USD (2554.34 TL), respectively. The decrease in drug prices in dollar terms between 2016 and 2018 is due to the depreciation of TL against USD. (USD / TL average rate was 3.02, 3.65 , and 4.82 in 2016, 2017, 2018, respectively.)

This study was conducted at the Department of Ophthalmology of Sakarya University Medical Education and Research Hospital. Prior approval from the Institutional Review Board was taken and written informed consent was obtained from each subject. The study was performed in adherence to the Declaration of Helsinki.

\section{STATISTICAL ANALYSIS}

Data were analyzed using SPSS (version 17.0, SPSS Inc., Chicago, IL, USA) and Microsoft Excel (Microsoft Corp., Redmond, WA, USA). Descriptive analysis was used to analyze the data and the numerical data were given as mean and standard deviation (SD). The Kolmogorov-Smirnov test was used for the analysis of the distribution of the normality, and while the parametric Student's t-test was used for the analysis of the normally distributed parameters, non-parametric ANOVA test and Post Hoc Tests (Tamhane) were used to analyze abnor- 
mally distributed data. $\mathrm{p}<0.05$ was considered statistically significant.

\section{RESULTS}

In total, data of 1525 patients were analyzed: $742(48.7 \%)$ of the patients were male and $783(51.3 \%)$ were female. The mean age of the patients was $66.17 \pm 10.36$ years in males and $65.36 \pm 9.95$ years in females, and there was not a statistically significant difference between them $(\mathrm{p}=0.118) .801$ patients $(52.5 \%)$ had DME, 395 (25.9\%) had neovascular AMD, and 329 (21.6\%) had RVO. The total number of intravitreal anti-VEGFs in 3 years was 5864 . The anti-VEGF numbers in 2016, 2017 and 2018 were 1997, 1801, 2066, respectively (Tab. 1). In total, 3 -year drug cost was 3,587,812.44 USD, and the distribution of drugs by years and drug type is summarized in Table 1.

During the 3-year follow-up, on average annually, ranibizumab (Lucentis) was applied 3.56 \pm 2.25 times (range 1-13), alfibercept (Eylea) was applied $3.31 \pm 2.16$ times (1-19), and IVD (Ozurdex) was applied $1.70 \pm 0.83$ times (range 1-4) There was no significant difference between the number of ranibizumab administered in DME and the number applied in AMD, but it was significantly higher than RVO. In addition, the average number of ranibizumab applied in AMD was significantly higher than RVO ( $p=0.249, p<0.05, p=0.045)$. There was no significant difference between the mean alfibercept number applied in DME and AMD, but DME was significantly higher than RVO. However, no significant difference was found between the mean alfibercept number applied in AMD and those applied in RVO ( $p=0.858, p=0.004, p=0.069)$. Intravitreal dexamethasone implant was used in patients with DME and RVO, and its use was significantly higher in patients with DME than in patients with RVO $(\mathrm{p}<0.05)$ (Tab. 2).

\section{DISCUSSION}

Vision loss due to retinal diseases such as DME, AMD, and RVO is increasing day by day and these diseases are a major part of visual loss [14-16]. Intravitreal anti-VEGF is one of the proven treatment modalities for reducing visual loss $[17,18]$. However, the high cost of anti-VEGF agents and their frequent repetition constitute a serious economic burden [9]. In this study, we aimed to investigate the economic burden of intravitreal anti-VEGF and IVD treatment for DME, AMD and RVO.

There are several studies analyzing the frequency and cost of anti-VEGF use in retinal diseases. In his study, Turpcu et al. compared the number and cost of intravitreal injections applied in DME, RVO and AMD. In AMD, an annual average of 5.6 times alfibercept and 5.3 times ranibizumab were performed while 4.5 times aflibercept and 5 times ranibizumab were performed in $\mathrm{RVO}$, and there was no significant difference in anti-VEGF usage numbers and prices. On average, $4.4 \pm 2.9$ times

\begin{tabular}{|l|c|c|c|c|c|c|c|c|}
\hline \multicolumn{7}{l}{ Table 1. Distribution and cost of intravitreal drugs by years } \\
\hline & $\mathbf{2 0 1 6}(\mathbf{n})$ & $\mathbf{2 0 1 7}(\mathbf{n})$ & $\mathbf{2 0 1 8}(\mathbf{n})$ & Total (n) & $\mathbf{2 0 1 6}$ (USD) & $\mathbf{2 0 1 7}$ (USD) & $\mathbf{2 0 1 8}$ (USD) & Total (USD) \\
\hline Lucentis & 1096 & 712 & 1056 & 2864 & $772,632.82$ & $458,941.54$ & $534,097.20$ & $1,765,671.56$ \\
\hline Eylea & 901 & 1089 & 1010 & 3000 & $534,483.94$ & $540,636.28$ & $420,168.40$ & $1,495,288.61$ \\
\hline Ozurdex & 121 & 150 & 292 & 563 & $80,672.54$ & $91,435.47$ & $154,744.20$ & $326,852.27$ \\
\hline Total & 1997 & 1801 & 2066 & 5864 & $1,387,789.30$ & $1,091,013.31$ & $1,109,009.80$ & $3,587,812.44$ \\
\hline
\end{tabular}

\begin{tabular}{|c|c|c|c|c|c|c|c|}
\hline & DME (Mean) & AMD (Mean) & RVO (Mean) & Total (Mean) & p (DME-AMD) & p (DME-RVO) & p (AMD-RVO) \\
\hline $\begin{array}{l}\text { Lucentis } \\
(\mathrm{n}=805)\end{array}$ & $3.79 \pm 2.30$ & $3.46 \pm 2.303$ & $2.91 \pm 1.84$ & $3.56 \pm 2.25$ & 0.249 & $<0.05$ & 0.045 \\
\hline $\begin{array}{l}\text { Eylea } \\
(\mathrm{n}=907)\end{array}$ & $3.46 \pm 2.12$ & $3.34 \pm 2.31$ & $2.90 \pm 1.94$ & $3.31 \pm 2.16$ & 0.858 & $<0.05$ & 0.069 \\
\hline $\begin{array}{l}\text { Ozurdex } \\
(\mathrm{n}=331)\end{array}$ & $1.80 \pm 0.82$ & - & $1.53 \pm 0.82$ & $1.70 \pm 0.83$ & - & $<0.05$ & - \\
\hline
\end{tabular}


ranibizumab was administered to DME patients in a year. The annual cost of treatment with ranibizumab in DME patients was lower than in RVO and AMD patients [11]. In our study, it was observed that the mean number of injections was lower and it is thought that this was due to long follow-up periods (3 years) and treatment protocol In their study, Johnson et al. found that the total direct health care cost per patient in patients treated with anti-VEGF was 14,725 euros in France, 10,927 euros in Spain and 9,647-13,759 pounds in the UK and $50-80 \%$ of this cost was the drug cost. They also reported that the most commonly used anti-VEGF agent was ranibizumab [19]. Drug prices in Turkey are cheaper than in Europe and anti-VEGF annual costs are significantly lower than the European countries. However, Turkey is a developing country and despite being cheaper in dollar, anti-VEGF agents are estimated to have a higher economic burden in the country.

In a study conducted by Hollingworth et al., the use of anti-VEGF agents (ranibizumab, bevacizu$\mathrm{mab}$ ) in various retinal diseases in the UK has been found to increase by $215 \%$ over a 5 -year period (2010/2011 to $2014 / 2015)$. In addition, it is estimated that there will be a 447 million pounds drug spending during the 2015/2016 period due to the intravitreal use of anti-VEGF agents [20]. Moreover, these expenditures are expected to increase with each passing day. In a study by Dakin et al., it was reported that the replacement of ranibizumab treatment for AMD patients with bevacizumab would save around 102 million pounds annually [21]. In another study, Hutton et al. reported that in the 10 -year period (2010-2020), approximately 18 billion dollars could be saved if all other anti-VEGF agents were replaced with bevacizumab [22]. In our study, it is seen that the number of patients with a sudden-VEGF need has increased over the years and the economic burden created by this situation also increases. We believe that the transition to bevacizumab will provide significant savings.

Bevacizumab is an anti-VEGF agent with proven efficacy in retinal diseases such as DME, AMD, RVO, despite the lack of FDA approval for intravitreal use (indication in colon cancer) $[6,23$, 24]. In addition, the cost of the intravitreal use of bevacizumab is quite inexpensive compared to other drugs [25]. In addition, many studies have proven that bevacizumab is more cost-effective in various retinal diseases than other anti-VEGF agents on the market $[10,21,26]$. Turkish Medicines and Medical Devices Agency has confirmed the intravitreal use of bevacizumab since 2019 in Turkey. In light of this information, considering that there will be a significant increase in the use of bevacizumab from 2019 with the recent decision by TMMDA, we think that the economic burden created by anti-VEGF drugs will significantly decrease.

Legal and ethical issues are also important in anti-VEGF preferences of ophthalmologists. Hollingworth et al. reported that physicians had to choose expensive agents because of the lack of legal basis [20]. With the decision taken by the TMMDA, we think that the legal problems ophthalmologists in Turkey go through will reduce, and they may feel more comfortable in their anti-VEGF preferences.

\section{CONCLUSION}

As a result, we think the financial burden created by anti-VEGF in Turkey will reduce in a serious sense, and the legal concerns of physicians will decrease thanks to the decision taken by the TMMDA. We intend to continue our current work in the following years. Thus, we will have the opportunity to evaluate the amount of money spent on anti-VEGF drugs and the savings provided.

\section{Acknowledgments}

We are grateful to Atilla Akgül, one of the staff of the Sakarya Provincial Health Directorate, for his contributions to the data collection.

\section{REFERENCES}

1. Bourne R, Stevens $G$, White $R$, et al. Causes of vision loss worldwide, 1990-2010: a systematic analysis. Lancet Glob Health. 2013; 1(6): e339-e349, doi: 10.1016/s2214-109x(13)70113-x, indexed in Pubmed: 25104599.

2. Li JO, Welchowski T, Schmid M et al. Retinal Diseases in Europe 2017. https://www.euretina.org/downloads/EURETINA_Retinal_Diseases.pdf (2017).

3. Cai $\mathrm{S}$, Bressler NM. Aflibercept, bevacizumab or ranibizumab for diabetic macular oedema: recent clinically relevant findings from DRCR.net Protocol T. Curr Opin Ophthalmol. 2017; 28(6): 636-643, doi: 10.1097/ICU.0000000000000424, indexed in Pubmed: 28837425.

4. Hanhart J, Rozenman Y. Comparison of Intravitreal Ranibizumab, Aflibercept, and Dexamethasone Implant after Bevacizumab Failure in Macular Edema Secondary to Retinal Vascular Occlusions. Ophthalmologica. 2017; 238(1-2): 110-118, doi: 10.1159/000473864, indexed in Pubmed: 28535531.

5. Kovach JL, Schwartz SG, Flynn HW, et al. Anti-VEGF Treatment Strategies for Wet AMD. J Ophthalmol. 2012; 2012: 786870, doi: 10.1155/2012/786870, indexed in Pubmed: 22523653.

6. Cai S, Bressler N. Aflibercept, bevacizumab or ranibizumab for diabetic macular oedema. Current Opinion in Ophthalmology. 2017; 28(6): 636-643, doi: 10.1097/icu.0000000000000424. 
7. Chakravarthy U, Harding SP, Rogers CA, et al. IVAN Study Investigators. Ranibizumab versus bevacizumab to treat neovascular age-related macular degeneration: one-year findings from the IVAN randomized trial. Ophthalmology. 2012; 119(7): 1399-1411, doi: 10.1016/j. ophtha.2012.04.015, indexed in Pubmed: 22578446.

8. Erie JC, Barkmeier AJ, Hodge DO, et al. High Variation of Intravitreal Injection Rates and Medicare Anti-Vascular Endothelial Growth Factor Payments per Injection in the United States. Ophthalmology. 2016; 123(6): 1257-1262, doi: 10.1016/j.ophtha.2016.02.015, indexed in Pubmed: 26976701.

9. Baker-Schena L. Expensive Drugs. Eye Net Mag. 2017: 39-44.

10. Ross EL, Hutton DW, Stein JD, et al. Diabetic Retinopathy Clinical Research Network. Cost-effectiveness of Aflibercept, Bevacizumab, and Ranibizumab for Diabetic Macular Edema Treatment: Analysis From the Diabetic Retinopathy Clinical Research Network Comparative Effectiveness Trial. JAMA Ophthalmol. 2016; 134(8): 888-896, doi: 10.1001/ jamaophthalmol.2016.1669, indexed in Pubmed: 27280850.

11. Turpcu A, Wilson K, Huang A et al. Injection frequency and costs of anti-vegf treatments for neovascular age-related macular degeneration, retinal vein occlusion, and diabetic macular Eedema. Value Heal 2015; 18(3): A180. http://linkinghub.elsevier.com/retrieve/pii/ S1098301515011018.

12. Sosyal Güvenlik Kurumu. Sosyal Güvenlik Kurumu Sağlık Uygulama Tebliği. Sosyal Güvenlik Kurumu Sağlk Uygulama Tebliği. 2018. http:// www.sgk.gov.tr/wps/portal/sgk/tr/kurumsal/merkez-teskilati/ ana_hizmet_birimleri/gss_genel_mudurlugu/anasayfa_duyurular/ guncel_sut_28122018.

13. Schmidt-Erfurth U, Chong V, Loewenstein A, et al. European Society of Retina Specialists. Guidelines for the management of neovascular age-related macular degeneration by the European Society of Retina Specialists (EURETINA). Br J Ophthalmol. 2014; 98(9): 1144-1167, doi: 10.1136/ bjophthalmol-2014-305702, indexed in Pubmed: 25136079.

14. Lee R, Wong TY, Sabanayagam C. Epidemiology of diabetic retinopathy, diabetic macular edema and related vision loss. Eye Vis (Lond). 2015; 2: 17, doi: 10.1186/s40662-015-0026-2, indexed in Pubmed: 26605370.

15. Wong WL, Su X, Li X, et al. Global prevalence of age-related macular degeneration and disease burden projection for 2020 and 2040: a systematic review and meta-analysis. Lancet Glob Health. 2014; 2(2): e106-e116, doi: 10.1016/S2214-109X(13)70145-1, indexed in Pubmed: 25104651

16. Flaxman $S$, Bourne R, Resnikoff $S$, et al. Global causes of blindness and distance vision impairment 1990-2020: a systematic review and meta-analysis. The Lancet Global Health. 2017; 5(12): e1221-e1234, doi: 10.1016/s2214-109x(17)30393-5, indexed in Pubmed: 29032195.

17. Wecker T, Ehlken C, Bühler A, et al. Five-year visual acuity outcomes and injection patterns in patients with pro-re-nata treatments for
AMD, DME, RVO and myopic CNV. Br J Ophthalmol. 2017; 101(3): 353-359, doi: 10.1136/bjophthalmol-2016-308668, indexed in Pubmed: 27215744.

18. Ou WC, Brown DM, Payne JF, et al. Relationship Between Visual Acuity and Retinal Thickness During Anti-Vascular Endothelial Growth Factor Therapy for Retinal Diseases. Am J Ophthalmol. 2017; 180: 8-17, doi: 10.1016/j.ajo.2017.05.014, indexed in Pubmed: 28549848

19. Johnson MK, Lara N. PSS18 Direct Economic Burden of Regular Intravitreal Injections for the Treatment of Retina Diseases in Three European Countries. Value Heal. Elsevier Inc. 2011; 14(7): A505. https:// linkinghub.elsevier.com/retrieve/pii/S1098301511030452.

20. Hollingworth $\mathrm{W}$, Jones $\mathrm{T}$, Reeves $\mathrm{BC}$, et al. A longitudinal study to assess the frequency and cost of antivascular endothelial therapy, and inequalities in access, in England between 2005 and 2015. BMJ Open. 2017; 7(10): e018289, doi: 10.1136/bmjopen-2017-018289, indexed in Pubmed: 29061629.

21. Dakin HA, Wordsworth S, Rogers CA, et al. IVAN Study Investigators. Cost-effectiveness of ranibizumab and bevacizumab for age-related macular degeneration: 2-year findings from the IVAN randomised trial. BMJ Open. 2014; 4(7): e005094, doi: 10.1136/bmjopen-2014-005094, indexed in Pubmed: 25079928.

22. Hutton D, Newman-Casey PA, Tavag M, et al. Switching to less expensive blindness drug could save medicare part B $\$ 18$ billion over a ten-year period. Health Aff (Millwood). 2014; 33(6): 931-939, doi: 10.1377/hlthaff.2013.0832, indexed in Pubmed: 24889941.

23. Narayanan R, Panchal B, Das T, et al. MARVEL study group. A randomised, double-masked, controlled study of the efficacy and safety of intravitreal bevacizumab versus ranibizumab in the treatment of macular oedema due to branch retinal vein occlusion: MARVEL Report No. 1. Br J Ophthalmol. 2015; 99(7): 954-959, doi: 10.1136/ bjophthalmol-2014-306543, indexed in Pubmed: 25631483.

24. Martin DF, Maguire MG, Fine SL, et al. Comparison of Age-related Macular Degeneration Treatments Trials (CATT) Research Group. Ranibizumab and bevacizumab for treatment of neovascular agerelated macular degeneration: two-year results. Ophthalmology. 2012; 119(7): 1388-1398, doi: 10.1016/j.ophtha.2012.03.053, indexed in Pubmed: 22555112.

25. Raftery J, Clegg A, Jones J, et al. Ranibizumab (Lucentis) versus bevacizumab (Avastin): modelling cost effectiveness. Br J Ophthalmol. 2007; 91(9): 1244-1246, doi: 10.1136/bjo.2007.116616, indexed in Pubmed: 17431015.

26. van Asten F, Michels CTJ, Hoyng CB, et al. The cost-effectiveness of bevacizumab, ranibizumab and aflibercept for the treatment of agerelated macular degeneration-A cost-effectiveness analysis from a societal perspective. PLoS One. 2018; 13(5): e0197670, doi: 10.1371/ journal.pone.0197670, indexed in Pubmed: 29772018. 